\title{
Hybrid Image Compression Method using ANN and DWT
}

\author{
Yogita Sawant \\ D.K.T.E'S Text.\& Engg. Institute \\ Ichalkaranji. India 416115
}

\author{
L. S. Admuthe \\ D.K.T.E'S Text.\& Engg .Institute \\ Ichalkaranji, India 416115
}

\begin{abstract}
Transmission of image and video requires particularly large amount of bandwidth and storage space. Image compression technology is useful to overcome these problems. Various method of compression like transform coding, predictive coding, bit plane coding are available and are used efficiently. Compression systems based on hybrid coding which combines the advantages of different methods of image compression have also being developed over the last few years. Hybrid coding of images, deals with combining various approaches to enhance the individual methods and achieve better quality reconstructed images with higher compression ratio. In this paper, a hybrid approach to image compression is discussed. A compression method using neural-network and discrete wavelet transform is presented here. This scheme combines the high compression ratio of Self organizing map neural network with the good recreation property of discrete wavelet transform (DWT).The performance of proposed method is compared with the available SOFM NN based compression technique considering standard images.
\end{abstract}

Keywords: Artificial Neural Networks, Image compression, Self organizing feature map neural network (SOFM-NN), DWT.

\section{INTRODUCTION}

The main objective of image compression is to decrease the redundancy of the image data which helps in increasing the capacity of storage and efficient transmission. Image compression aids in decreasing the size in bytes of a digital image without degrading the quality of the image to an undesirable level. There are two classifications in image compression: lossless and lossy compression. The reduction in file size allows more images to be stored in a given amount of disk or memory space. This supports in decreasing the time required for the image to send or download from internet.

Apart from the existing technology on image compression represented by series of JPEG, MPEG and H.26x standards, technology such as neural networks and genetic algorithms are being developed to explore the future of image coding. Successful applications of neural networks with back propagation algorithm have now become well established and other aspects of neural network involvement in various technologies are being studied [1]. Research activities on neural networks for image compression do exist in many types of networks such as -Multi Layer Perceptron (MLP) [2][3], Hopfield [14], Self-Organizing Map (SOM), Learning Vector Quantization ( LVQ)[5].

Image compression using Wavelet Transforms is a powerful method of lossy compression that is preferred by scientists to store the images at higher compression ratios[6]. Wavelet facilitates image compression using less storage space with more details of the image. Wavelet Image Processing facilitates computers to accumulate an image in many scales of resolutions, decomposing an image into various levels and types of details. This paper implements a hybrid approach for compression of gray scale images using self organizing map (SOM) neural network and discrete wavelet transform. The compression achieved by SOM neural network is higher as compared to other networks. But it is further increased using wavelet transform which will compress the code vectors of SOM. The performance is evaluated using various performance measures.

\section{COMPRESSION BY NEURAL NETWORK}

Artificial neural network models are specified by network topology (architecture) and learning algorithms [1],[2]. Network topology describes the way in which the neurons (basic processing unit) are interconnected and the way in which they receive input and output. Learning algorithms specify an initial set of weights and indicate how to adapt them during learning in order to improve network performance. A neural network has number of simple computational units, called neurons which are interconnected to form a network, to perform complex computational tasks. Because of its parallel architecture, Artificial Neural Networks (ANNs)have been applied to image compression problems, due to their superiority over traditional methods when dealing with noisy or incomplete data[7][11]. ANNs seem to be well suited to image compression, as they have the ability to preprocess input patterns to produce simpler patterns with fewer components. This compressed information preserves the full information obtained from the external environment. Neural Networks based compression gives security of the data to be compressed. Many different training algorithms and architectures have been used. Different types of Artificial Neural Networks have been trained to perform image Compression. In image compression technique, the compression is achieved by training a neural network with the image and then using the weights and the coefficients from the hidden layer as the data to recreate the image.

\subsubsection{Self Organizing Feature Map Neural Network}

Out of three categories of neural network (NN) architectures, namely Feed forward networks, Feed Back networks and Recurrent networks, the self organizing neural net belongs to last one that is recurrent, or competitive or unsupervised networks. A number of researchers have successfully used the Self Organizing Feature map (SOFM) algorithm to image compression [9][13]. SOFM's have two properties. First, it quantizes the input space like any other vector quantization method, which constitutes a lossy 
compression of the image. Then, the topology preserving property of SOFM, coupled with the hypothesis that consecutive blocks in the image will often be similar, and to a differential entropic coder, constitutes a second (non-lossy) compression of the information. This means SOFM neural nets can give better compression ratios than other networks [4].

SOFM is realized by a two-layer network, as shown in Fig.1.The first layer is the input layer or fan-out layer with input neurons and the second layer is the output or competitive layer. The two layers are completely connected. An input vector $x \in$ $R^{k}$ when applied to the input layer, is distributed to each of the $(m \times n)$ output nodes $\mathrm{O}_{\mathrm{k}}$ in the competitive layer. Each node in this layer is connected to all nodes in the input layer; hence, it has a weight vector prototype $w_{i j}$ attached to it. The node in the competitive layer are cluster centers while grouping the data points.
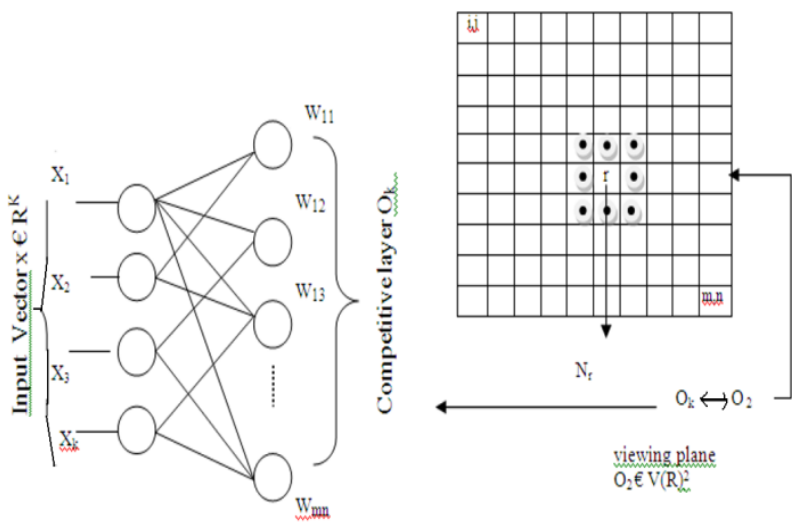

Figure 1. SOFM network architecture

In SOFM, each data from data set recognizes themselves by competing for representation. The weight vectors initialization is the starting process of SOFM mapping[4]. Then the sample vector from data set is randomly selected and the map of weight vectors is searched to find which weight best represents that sample. Each weight vector has neighboring weights that are close to it. The weight that is chosen is rewarded by being able to become more like that randomly selected sample vector. The neighbors of that weights are also rewarded by being able to become more like the chosen sample vector. From this step the number of neighbors and how much each weight can learn decreases over time. This whole process is repeated a large number of times, usually more than 1000 times.

In sum, learning occurs in several steps and over much iteration:

1. Each node's weights are initialized.

2. A vector is chosen at random from the set of training data.

3. Every node is examined to calculate which one's weights are most like the input vector. The winning node is commonly known as the Best Matching Unit (BMU).

4. Then the neighborhood of the BMU (r) is calculated. The amount of neighbors decreases over time.

5. The winning weight is rewarded with becoming more like the sample vector. The neighbors also become more like the sample vector. The closer a node is to the BMU, the more its weights get altered and the farther away the neighbor is from the BMU, the less it learns.

6. Repeat step 2 for $\mathrm{N}$ iterations.
After all vectors from input dataset are clustered, the BMUs are used to represent the blocks of the image as shown in figure 1 .

\section{COMPRESSION BY WAVELET TRANSFORM}

Wavelet transform is the representation of any arbitrary signal $x(t)$ as a weighted sum of a set of basis functions called mother wavelet $\psi$,by dilations ( $\tau$ ) and translations (s) given as,

$\psi_{\mathrm{S}, \tau}(\mathrm{t})=\frac{1}{\sqrt{\mathrm{s}}} \psi\left(\frac{\mathrm{t}-\tau}{\mathrm{s}}\right)$

Low frequencies are examined with low temporal resolution while high frequencies with more temporal resolution. A wavelet transform combines both low pass and high pass filtering in spectral decomposition of signals. A wavelet transform combines both low pass and high pass filtering in spectral decomposition of signals. In wavelet transform based compression method, the original image or data matrix of size say $\mathrm{M} \times \mathrm{N}$ is first decomposed in the horizontal direction into two halves by the low pass filter $\mathrm{L}_{\mathrm{h}}(\mathrm{z})$ and high pass filter $\mathrm{H}_{\mathrm{h}}(\mathrm{z})$. The output of each filter is then down sampled with a factor of 2. Then the output is further partitioned in the vertical direction into two halves by the same filters. The output of each filter is again down sampled with a factor of 2 , which yields four image bands LL, LH, HL and HH of the first scale as shown in Fig.3. LL, LH and HL are termed as low-frequency bands and HH band is called as high frequency bands[12],[15].

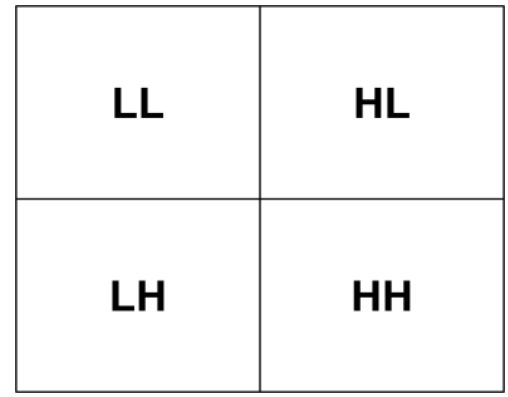

Figure 2: Two level wavelet decomposition

After wavelet decomposition, the lower frequencies bands are adopted as the bands with noteworthy features. Hence these bands are collected together to form the wavelet coefficients. Then the coefficients are quantized by vector quantization technique with respect to an expected compression rate. The obtained coefficients are capable of reproducing all the image data values at the time of reconstruction by inverse wavelet transform process. In this work of hybrid compression, this property of wavelet transform has been used to compress the code vectors generated by SOFM neural network.

\section{COMPRESSION BY HYBRID METHOD}

The hybrid approach combines the advantage of both SOFM neural network and wavelet transform to enhance the performance of the compression[16],[17]. The SOFM neural net will be used to obtain the code vectors and then those code vectors are again compressed by DWT This method will be implemented with the help of following steps:-

a) Pre processing of image. And converting image data blocks into vectors.

b) Training of SOFM neural network to generate code vectors. 
c) Apply wavelet transform to reduce the size of code vector.

d) Encoding of wavelet coefficients.

e) Decompression of image in decoder using codebook \& inverse wavelet transform.

f) The quality analysis of the decompressed images is carried out using performance parameters.

\section{IMPLEMENTATION AND RESULTS}

In order to evaluate the performance of the proposed hybrid approach of image compression, standard images like Lena and Cameraman $(256 \times 256)$ are used. The work is implemented using MATLAB. Performance of the hybrid approach is evaluated using various measures. They are used to judge the quality of image compression. This work uses statistical parameters like compression ratio(CR), Peak Signal-to-Noise Ratio (PSNR) and some the co-relational based quality measures like $\mathrm{AD}$ (average difference) \& IF(Image fidelity) [18]. These measures look at correlation features between the pixels of original and reconstructed image. The experiments are carried out with the number of clusters as 64, 128 and 256 etc. The method first uses SOFM neural network to generate the desired codebook. The image is blocked $(4 \times 4)$ and is fed the network for training up to 100 epoch to generate the code vectors. Each code vector will be of 16 elements. To reduce the size of each vector Wavelet transform is used. The DWT will reduce the size of the code vector from 16 elements to 4 elements. The mother wavelet used for DWT was 'db1'.

The experimentation with codebook generated using same image was carried out and it has been observed that proposed method gives higher compression ratio over SOFM-neural network(NN) method. The reconstructed image quality is also retained. Following tables shows the results obtained and comparisons of both approaches using above mentioned quality measures. Figure 3.and table1 shows results of compression using SOFM neural network only. Using hybrid method, for a $256 \times 256$ Lena image for example, if the block is chosen is $4 \times 4$ and number of clusters or code vectors equal to 128 , the PSNR of $28.83 \mathrm{~dB}$ is obtained. The PSNR for same configuration, but using SOFM-NN method is about $27.12 \mathrm{~dB}$. If the number of code vectors are increased the PSNR value increases for both methods. The important thing is compression ratio with hybrid approach is improved
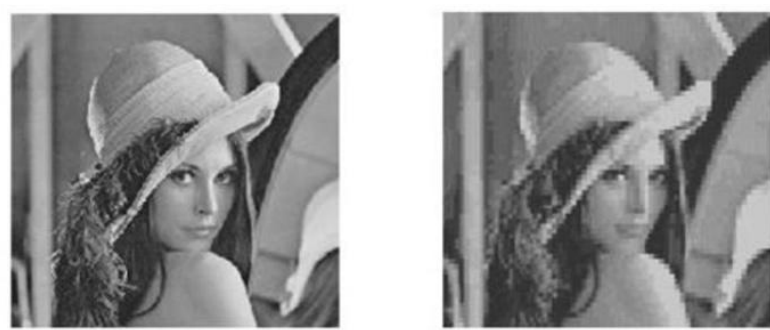

Figure 3. Standard Image Lena_gray.tif (left) Original image (right) Decompressed image by SOFM-NN

Table.1 Results for Image Lena, using SOFM- NN

\begin{tabular}{|l|c|c|c|c|}
\hline $\begin{array}{l}\text { Image } \\
\text { Block } \\
\text { size }\end{array}$ & $\begin{array}{c}\text { PSNR } \\
\text { dB }\end{array}$ & IF & AD & CR \\
\hline $2 \times 2$ & 27.33 & 0.9970 & 0.0364 & $128: 1$ \\
\hline $4 \times 4$ & 26.81 & 0.9953 & 0.1133 & $64: 1$ \\
\hline $4 \times 4$ & 27.12 & 0.9911 & -0.0534 & $32: 1$ \\
\hline $4 \times 4$ & 28.68 & 0.9917 & -0.0542 & $16: 1$ \\
\hline $8 \times 8$ & 22.46 & 0.9839 & -0.0123 & $16: 1$ \\
\hline $8 \times 8$ & 22.87 & 0.9853 & -0.0541 & $8: 1$ \\
\hline $8 \times 8$ & 23.88 & 0.9861 & -0.0534 & $4: 1$ \\
\hline
\end{tabular}

Figure 4 and Table 2 shows results of compression using hybrid method.
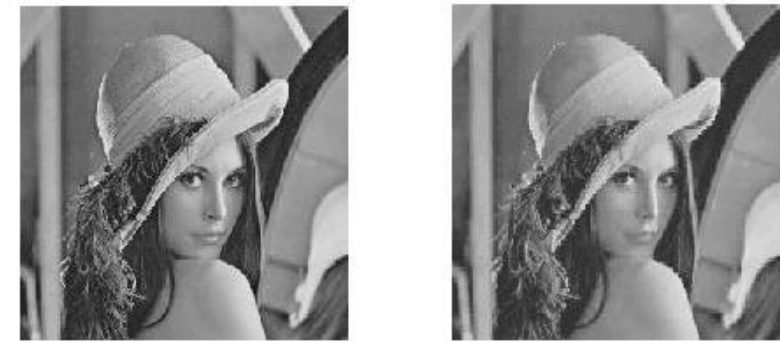

Figure 4. Image -Lena_gray.tif, (left) Original image (right)Decompressed image using hybrid method with PSNR=28.21 $\mathrm{dB}$.

Table 2. Results of hybrid ANN -DWT method

\begin{tabular}{|c|l|c|c|c|}
\hline $\begin{array}{l}\text { Image } \\
\text { Block } \\
\text { size }\end{array}$ & PSNR & IF & AD & CR \\
\hline $2 \times 2$ & 32.15 & 0.9969 & -2.4851 & $256: 1$ \\
\hline $4 \times 4$ & 26.21 & 0.9929 & -4.1854 & $128: 1$ \\
\hline $4 \times 4$ & 27.21 & 0.9958 & -3.8413 & $64: 1$ \\
\hline $4 \times 4$ & 28.83 & 0.9964 & -3.6959 & $32: 1$ \\
\hline $8 \times 8$ & 25.55 & 0.9920 & -5.8840 & $32: 1$ \\
\hline $8 \times 8$ & 26.01 & 0.9932 & -5.3699 & $16: 1$ \\
\hline
\end{tabular}

\section{CONCLUSIONS}

In this paper image compression using hybrid method of ANN and DWT is discussed. The compression ratio using this method is almost doubled. The PSNR for Lena image by proposed method is $1-2 \mathrm{~dB}$ more as compared to SOFM neural network method. It has been observed that if the block size is reduced then PSNR and CR increase. But the value of average difference is increased. Thus the combination with discrete wavelet transform can provide higher compression ratio along with slightly higher PSNR values than SOFM Neural network based method.

The neural networks like SOFM have capability to compress the images at higher compression rates. Wavelet transform shows faithful reconstruction of compressed vectors using approximation coefficients from LL sub band. The hybridization using both these benefits shows significant improvements in the performance of the compression algorithm which is used to enhance the performance of ANN based compression methods. The future approach can make the use of some statistical parameters like mean and standard deviation to select the blocks of image or region of interest, the quality measures can be further improved.

\section{REFERENCES}

[1] Cottrell et.al."Learning internal representation from grayscale images:, An example of extensional programming." In Proceedings of the Ninth Conference of the Cognitive Science Society, 1988.

[2] R.D. Dony, S. Haykin, "Neural network approaches to image compression", Proc. IEEE, proc. Vol. 83, No. 2, Feb. 1995, pp 288-303.

[3] A. Namphol, S. Chin, M. Arozullah, "Image compression with a hierarchical neural network", IEEE Trans. 
Aerospace Electronic Systems, Vol 32, No.1, January 1996, pp326-337.

[4] Christophe Amerijckx et.al, "Image Compression by Selforganized Kohonen maps", IEEE Trans. on Neural Networks, Vol.9, No.3, May 1998.

[5]J. Jiang, "Image Compression with neural networks - A survey", Signal Processing: Image communication, Elsevier Science B.V., Vol.14, 1999, pp 737-760.

[6] Antonini, M.; Barlaud, M.; Mathieu, P.; Daubechies, I, "Image coding using wavelet transform" IEEE Trans. on Image processing Vol.2,2002,pp 205-220.

[7] S. Anna Durai, E. Anna Saro, "Image Compression with Back- Propagation Neural Network using Cumulative Distribution Function", International Journal for Applied Science and Engg. Technology 2006.

[8]S.S.Panda et.al, Image compression using back propagation neural network", IJESAT 2012 Vol 2.

[9] Ren-Jean Liou, et.al "Quadtree Image Compression Using Sub-Band DCT Features and Kohonen Neural Networks by" IEEE Proc. 2008 .

[10]Y. Linde, A. Buzo, and R. M. Gray, "An algorithm for vector quantizer design," IEEE Trans. Commun., vol.COMM-28, pp. 84-95, Jan. 1980.

[11]Ivan Vilovic' “An Experience in Image Compression Using Neural Networks", 48th International Symposium ELMAR-2006, 07-09 June 2006.
[12] Amjan Shaik, et.al, "Empirical Analysis of Image Compression through Wave Transform and Neural Network". IJCSIT Vol. 22011

[13] G.Boopathy.et.al, "Implementation of Vector Quantization for Image Compression - A Survey" Global Journal of Computer Science and Technology Vol. 10 Issue 3 April 2010.

[14] Robina Ashraf et.al "Adaptive Architecture Neural Nets for Medical Image Compression” IEEE Proceeding, 2006.

[15] Rafael C. Gonzalez, Richard E. Woods, Digital Image Processing, Third Edition, Pearson Education 2008.

[16] G.Boopathi,"An Image Compression Approach using Wavelet Transform and Modified Self Organizing Map", IJCSI International Journal of Computer Science Issues, Vol. 8, Issue 5, No 2, September 2011.

[17] Chaabouni,I.“An improved image compression approach with combined wavelet and self organizing maps" Electro technical Conference (MELECON), Mar 2012 16th IEEE Mediterranean.

[18] Marta Mrak, et.al "Picture quality measures in image compression systems". EUROCON, Ljuijana, Slovenia. 2003.

[19] A.K. Krishnamurthy et al., "Neural Networks for VQ of speech and Images", IEEE journal On selected areas in comm., Vol.8, No.8,sept.2006

[20] Dandawate et al. "Neuro-Wavelet based vector quantizer design for image compression", Indian Journal of Science and Technology Vol.2 No. 10 Oct 2009. 